\title{
On Some Properties of Universal Sigma-Finite Measures Associated with a Remarkable Class of Submartingales
}

\author{
by \\ Joseph NAJNUdel and Ashkan NikeghBALI
}

\begin{abstract}
In a previous work, we associated with any submartingale $X$ of class $(\Sigma)$, defined on a filtered probability space $\left(\Omega, \mathcal{F},\left(\mathcal{F}_{t}\right)_{t \geq 0}, \mathbb{P}\right)$ satisfying some technical conditions, a $\sigma$-finite measure $\mathcal{Q}$ on $(\Omega, \mathcal{F})$ such that for all $t \geq 0$, and for all events $\Lambda_{t} \in \mathcal{F}_{t}$,

$$
\mathcal{Q}\left[\Lambda_{t}, g \leq t\right]=\mathbb{E}_{\mathbb{P}}\left[\mathbb{1}_{\Lambda_{t}} X_{t}\right]
$$

where $g$ is the last hitting time of zero by the process $X$. In this paper we establish some remarkable properties of this measure from which we also deduce a universal class of penalization results of the probability measure $\mathbb{P}$ with respect to a large class of functionals. The measure $\mathcal{Q}$ appears to be the unifying object in these problems.
\end{abstract}

2010 Mathematics Subject Classification: 60G07, 60G17, 60G44.

Keywords: martingale, submartingale, penalization.

\section{Notation}

In this paper, $\left(\Omega, \mathcal{F},\left(\mathcal{F}_{t}\right)_{t>0}, \mathbb{P}\right)$ will denote a filtered probability space; $\mathcal{C}\left(\mathbb{R}_{+}, \mathbb{R}\right)$ is the space of continuous functions from $\mathbb{R}_{+}$to $\mathbb{R} \cdot \mathcal{D}\left(\mathbb{R}_{+}, \mathbb{R}\right)$ is the space of càdlàg functions from $\mathbb{R}_{+}$to $\mathbb{R}$. If $Y$ is a random variable, we denote by $\mathbb{P}[Y]$ or $\mathbb{E}_{\mathbb{P}}[Y]$ the expectation of $X$ with respect to $\mathbb{P}$. If $\left(A_{t}\right)_{t \geq 0}$ is an increasing process, as usual, the increasing limit of $A_{t}$, as $t \rightarrow \infty$, is denoted $A_{\infty}$.

\section{§1. Introduction}

In a paper by Madan, Roynette and Yor [7], and a set of lectures by Bentata and Yor [3], the authors prove that if $\left(M_{t}\right)_{t \geq 0}$ is a continuous nonnegative local

Communicated by T. Kumagai. Received December 18, 2009. Revised October 13, 2010.

J. Najnudel, A. Nikeghbali: Institut für Mathematik, Universität Zürich,

Winterthurerstrasse 190, 8057 Zürich, Switzerland;

e-mail: joseph.najnudel@math.uzh.ch, ashkan.nikeghbali@math.uzh.ch 
martingale defined on a filtered probability space $\left(\Omega, \mathcal{F},\left(\mathcal{F}_{t}\right)_{t \geq 0}, \mathbb{P}\right)$ satisfying the usual assumptions, and such that $\lim _{t \rightarrow \infty} M_{t}=0$, then for any $K \geq 0$,

$$
K \mathbb{P}\left[F_{t} \mathbb{1}_{g_{K} \leq t}\right]=\mathbb{P}\left[F_{t}\left(K-M_{t}\right)_{+}\right]
$$

where $g_{K}=\sup \left\{t \geq 0: M_{t}=K\right\}$. The formula (1.1), which represents the price of a European put option in terms of the probability distribution of some last passage time, gives, in a particular case, a positive answer to the following problem, also stated in [3] and [7]: for which submartingales $X$ can we find a $\sigma$-finite measure $\mathcal{Q}$ and the end of an optional set $g$ such that

$$
\mathcal{Q}\left[F_{t} \mathbb{1}_{g \leq t}\right]=\mathbb{P}\left[F_{t} X_{t}\right] ?
$$

This problem was previously encountered in the literature in different situations. In [2], Azéma and Yor prove that for any continuous and uniformly integrable martingale $M,(1.2)$ holds for $X_{t}=\left|M_{t}\right|, \mathcal{Q}=\left|M_{\infty}\right| \mathbb{P}$ and $g=\sup \left\{t \geq 0: M_{t}=0\right\}$, or equivalently

$$
\left|M_{t}\right|=\mathbb{P}\left[\left|M_{\infty}\right| \mathbb{1}_{g \leq t} \mid \mathcal{F}_{t}\right] .
$$

Here again the measure $\mathcal{Q}$ is finite. A particular case where the measure $\mathcal{Q}$ is not finite was obtained by Najnudel, Roynette and Yor in their study of Brownian penalizations (see [11]). For example, they prove the existence of the measure $\mathcal{Q}$ when $X_{t}=\left|W_{t}\right|$ is the absolute value of the standard Brownian motion. In this case, the measure $\mathcal{Q}$ is not finite but only $\sigma$-finite and is singular with respect to the Wiener measure: it satisfies $\mathcal{Q}(g=\infty)=0$, where $g=\sup \left\{t \geq 0: W_{t}=0\right\}$. Similarly Yano, Yano and Yor [16] have obtained the existence of the measure $\mathcal{Q}$ in the case where $X_{t}=\left|Y_{t}\right|^{\alpha-1}$, with $Y$ some symmetric Lévy stable process. Now, apart from its path decomposition obtained in [11], the existence of $\mathcal{Q}$ in all the examples cited above is a consequence of a general result proved by the authors of the present paper in [10]. The relevant class of submartingales is called $(\Sigma)$, it was first introduced by Yor in [17] and some of its main properties were further studied in [12]. Let us recall its definition.

Definition $1.1([12,17])$. Let $\left(\Omega, \mathcal{F},\left(\mathcal{F}_{t}\right)_{t \geq 0}, \mathbb{P}\right)$ be a filtered probability space. A nonnegative submartingale (resp. local submartingale) $\left(X_{t}\right)_{t \geq 0}$ is of class $(\Sigma)$ if it can be decomposed as $X_{t}=N_{t}+A_{t}$ where $\left(N_{t}\right)_{t \geq 0}$ and $\left(A_{t}\right)_{t \geq 0}$ are $\left(\mathcal{F}_{t}\right)_{t \geq 0^{-}}$ adapted processes satisfying the following assumptions:

- $\left(N_{t}\right)_{t \geq 0}$ is a càdlàg martingale (resp. local martingale).

- $\left(A_{t}\right)_{t \geq 0}$ is a continuous increasing process with $A_{0}=0$.

- The measure $\left(d A_{t}\right)$ is carried by the set $\left\{t \geq 0: X_{t}=0\right\}$. 
Note that a process of class $(\Sigma)$ is "almost" a martingale: outside the zeros of $X$, the process $A$ does not increase. In fact many processes one often encounters fall into this class, e.g. $X_{t}=\left|M_{t}\right|$ where $\left(M_{t}\right)_{t \geq 0}$ is a continuous local martingale, $X_{t}=\left(M_{t}-K\right)_{+}$where $\left(M_{t}\right)_{t \geq 0}$ is a càdlàg local martingale with only positive jumps and $K \in \mathbb{R}$ is a constant, $X_{t}=S_{t}-M_{t}$ where $\left(M_{t}\right)_{t \geq 0}$ is a local martingale with only negative jumps and $S_{t}=\sup _{u \leq t} M_{u}$. Other remarkable families of examples consist of a large class of recurrent diffusions on natural scale (such as some powers of Bessel processes of dimension $\delta \in(0,2)$, see [10]) or of functions of a symmetric Lévy process; in these cases, $A_{t}$ is the local time of the diffusion process or of the Lévy process.

Note that in the case where $A_{\infty}=\infty \mathbb{P}$-almost surely (this condition holds if $\left(X_{t}\right)_{t \geq 0}$ is a reflected Brownian motion), and $\left(\Omega, \mathcal{F},\left(\mathcal{F}_{t}\right)_{t \geq 0}, \mathbb{P}\right)$ satisfies the usual conditions, the measure $\mathcal{Q}$ cannot exist: otherwise, we would have, for all $t \geq 0$,

$$
\mathbb{P}\left[X_{t}\right]=\mathbb{P}\left[X_{t} \mathbb{1}_{g>t}\right]=\mathcal{Q}[g \leq t, g>t]=0
$$

since the event $\{g>t\}$ is $\mathbb{P}$-almost sure, and so in $\mathcal{F}_{t}$. Hence, $X$ would be indistinguishable from zero, which contradicts the fact that $A_{\infty}=\infty$. This explains why the usual conditions are not assumed in this paper. On the other hand, we also encounter some problems if we do not complete the probability spaces: for example, if $\Omega=\mathcal{C}\left(\mathbb{R}_{+}, \mathbb{R}\right), \mathcal{F}_{t}$ is the $\sigma$-algebra generated by the canonical process $X$ up to time $t$, and $\mathbb{P}$ is Wiener measure, then there does not exist a càdlàg $\left(\mathcal{F}_{t}\right)_{t \geq 0}$-adapted version of the local time which is well-defined everywhere (and not only $\mathbb{P}$-almost surely), as is explained for instance in [8]. In order to avoid also this technical problem, we assume that the filtration satisfies some particular conditions, intermediate between right-continuity and the usual conditions. These assumptions, called "natural conditions", were first introduced by Bichteler in [5] (p. 36), and then rediscovered in [8] (there they are also called N-usual conditions) where it is proved that most of the properties which generally hold under the usual conditions remain valid under the natural conditions (for example, existence of càdlàg versions of martingales, the Doob-Meyer decomposition, the début theorem, etc.). Let us recall here the definition.

Definition 1.2. A filtered probability space $\left(\Omega, \mathcal{F},\left(\mathcal{F}_{t}\right)_{t \geq 0}, \mathbb{P}\right)$ satisfies the natural conditions if the following two assumptions hold:

- The filtration $\left(\mathcal{F}_{t}\right)_{t \geq 0}$ is right-continuous.

- For all $t \geq 0$, and for every $\mathbb{P}$-negligible set $A \in \mathcal{F}_{t}$, all the subsets of $A$ are contained in $\mathcal{F}_{0}$. 
This definition is slightly different from the definitions given in [5] and [8] but one can easily check that it is equivalent. The natural enlargement of a filtered probability space can be defined by using the following proposition:

Proposition $1.1([8])$. Let $\left(\Omega, \mathcal{F},\left(\mathcal{F}_{t}\right)_{t \geq 0}, \mathbb{P}\right)$ be a filtered probability space. There exists a unique filtered probability space $\left(\Omega, \widetilde{\mathcal{F}},\left(\widetilde{\mathcal{F}}_{t}\right)_{t \geq 0}, \widetilde{\mathbb{P}}\right)$ (with the same set $\Omega$ ) such that:

- For all $t \geq 0, \widetilde{\mathcal{F}}_{t}$ contains $\mathcal{F}_{t}, \widetilde{\mathcal{F}}$ contains $\mathcal{F}$, and $\widetilde{\mathbb{P}}$ is an extension of $\mathbb{P}$.

- The space $\left(\Omega, \widetilde{\mathcal{F}},\left(\widetilde{\mathcal{F}}_{t}\right)_{t \geq 0}, \widetilde{\mathbb{P}}\right)$ satisfies the natural conditions.

- For any filtered probability space $\left(\Omega, \mathcal{F}^{\prime},\left(\mathcal{F}_{t}^{\prime}\right)_{t \geq 0}, \mathbb{P}^{\prime}\right)$ satisfying the two items above, $\mathcal{F}_{t}^{\prime}$ contains $\widetilde{\mathcal{F}}_{t}$ for all $t \geq 0, \mathcal{F}^{\prime}$ contains $\widetilde{\mathcal{F}}$, and $\mathbb{P}^{\prime}$ is an extension of $\widetilde{\mathbb{P}}$. The space $\left(\Omega, \widetilde{\mathcal{F}},\left(\widetilde{\mathcal{F}}_{t}\right)_{t \geq 0}, \widetilde{\mathbb{P}}\right)$ is called the natural enlargement of $\left(\Omega, \mathcal{F},\left(\mathcal{F}_{t}\right)_{t \geq 0}, \mathbb{P}\right)$.

Intuitively, the natural enlargement of a filtered probability space is its smallest extension which satisfies the natural conditions. We also introduce a class of filtered measurable spaces $\left(\Omega, \mathcal{F},\left(\mathcal{F}_{t}\right)_{t \geq 0}\right)$ such that any compatible family $\left(\mathbb{Q}_{t}\right)_{t \geq 0}$ of probability measures $\mathbb{Q}_{t}$ defined on $\mathcal{F}_{t}$ can be extended to a probability measure $\mathbb{Q}$ defined on $\mathcal{F}$.

Definition 1.3. Let $\left(\Omega, \mathcal{F},\left(\mathcal{F}_{t}\right)_{t \geq 0}\right)$ be a filtered measurable space such that $\mathcal{F}$ is the $\sigma$-algebra generated by $\mathcal{F}_{t}, t \geq 0: \mathcal{F}=\bigvee_{t \geq 0} \mathcal{F}_{t}$. We say that property $(\mathrm{P})$ holds if $\left(\mathcal{F}_{t}\right)_{t \geq 0}$ enjoys the following properties:

- For all $t \geq 0, \mathcal{F}_{t}$ is generated by a countable number of sets.

- For all $t \geq 0$, there exists a Polish space $\Omega_{t}$ and a surjective map $\pi_{t}$ from $\Omega$ to $\Omega_{t}$ such that $\mathcal{F}_{t}$ is the $\sigma$-algebra of the inverse images, under $\pi_{t}$, of Borel sets in $\Omega_{t}$, and for all $B \in \mathcal{F}_{t}$ and $\omega \in \Omega, \pi_{t}(\omega) \in \pi_{t}(B)$ implies $\omega \in B$

- If $\left(\omega_{n}\right)_{n \geq 0}$ is a sequence of elements of $\Omega$ such that for all $N \geq 0$,

$$
\bigcap_{n=0}^{N} A_{n}\left(\omega_{n}\right) \neq \emptyset,
$$

where $A_{n}\left(\omega_{n}\right)$ is the intersection of the sets in $\mathcal{F}_{n}$ containing $\omega_{n}$, then

$$
\bigcap_{n=0}^{\infty} A_{n}\left(\omega_{n}\right) \neq \emptyset .
$$

Remark. In the definition above, (P) stands for Parthasarathy since such conditions were introduced by him in [13, Chapter V, Section 4].

A fundamental example of a filtered measurable space $\left(\Omega, \mathcal{F},\left(\mathcal{F}_{t}\right)_{t \geq 0}\right)$ with property $(\mathrm{P})$ can be constructed as follows: We take $\Omega$ to be equal to $\mathcal{C}\left(\mathbb{R}_{+}, \mathbb{R}^{d}\right)$, 
the space of continuous functions from $\mathbb{R}_{+}$to $\mathbb{R}^{d}$, or $\mathcal{D}\left(\mathbb{R}_{+}, \mathbb{R}^{d}\right)$, the space of càdlàg functions from $\mathbb{R}_{+}$to $\mathbb{R}^{d}$ (for some $d \geq 1$ ), we define $\left(\mathcal{F}_{t}\right)_{t \geq 0}$ as the natural filtration of the canonical process, and we set $\mathcal{F}:=\bigvee_{t \geq 0} \mathcal{F}_{t}$. The combination of property $(\mathrm{P})$ and the natural conditions gives the following definition:

Definition 1.4. Let $\left(\Omega, \mathcal{F},\left(\mathcal{F}_{t}\right)_{t \geq 0}, \mathbb{P}\right)$ be a filtered probability space. We say that it has property $(\mathrm{NP})$ if is the natural enlargement of a filtered probability space $\left(\Omega, \mathcal{F}^{0},\left(\mathcal{F}_{t}^{0}\right)_{t \geq 0}, \mathbb{P}^{0}\right)$ such that the filtered measurable space $\left(\Omega, \mathcal{F}^{0},\left(\mathcal{F}_{t}^{0}\right)_{t \geq 0}\right)$ enjoys property $(\mathrm{P})$.

In [8] the following result on extension of probability measures is proved (in a slightly more general form):

Proposition 1.2. Let $\left(\Omega, \mathcal{F},\left(\mathcal{F}_{t}\right)_{t \geq 0}, \mathbb{P}\right)$ be a filtered probability space with property $(\mathrm{NP})$. Then $\mathcal{F}$ is the $\sigma$-algebra generated by $\left(\mathcal{F}_{t}\right)_{t \geq 0}$, and for any coherent family $\left(\mathbb{Q}_{t}\right)_{t \geq 0}$ of probability measures such that $\mathbb{Q}_{t}$ is defined on $\mathcal{F}_{t}$, and is absolutely continuous with respect to the restriction of $\mathbb{P}$ to $\mathcal{F}_{t}$, there exists a unique probability measure $\mathbb{Q}$ on $\mathcal{F}$ which coincides with $\mathbb{Q}_{t}$ on $\mathcal{F}_{t}$ for all $t \geq 0$.

By using all the results and definitions above, one can state rigorously the main result of [10] in its most general form:

Theorem 1.1. Let $\left(X_{t}\right)_{t \geq 0}$ be a submartingale of class $(\Sigma)$ (in particular $X_{t}$ is integrable for all $t \geq 0)$, defined on a filtered probability space $\left(\Omega, \mathcal{F},\left(\mathcal{F}_{t}\right)_{t \geq 0}, \mathbb{P}\right)$ with property $(\mathrm{NP})$ (in particular, $\left(\mathcal{F}_{t}\right)_{t \geq 0}$ satisfies the natural conditions and $\mathcal{F}$ is the $\sigma$-algebra generated by $\left.\mathcal{F}_{t}, t \geq 0\right)$. Then there exists a unique $\sigma$-finite measure $\mathcal{Q}$, defined on $(\Omega, \mathcal{F}, \mathbb{P})$, such that for $g:=\sup \left\{t \geq 0: X_{t}=0\right\}$ :

- $\mathcal{Q}[g=\infty]=0$.

- For all $t \geq 0$, and for all $\mathcal{F}_{t}$-measurable, bounded random variables $F_{t}$,

$$
\mathcal{Q}\left[F_{t} \mathbb{1}_{g \leq t}\right]=\mathbb{P}\left[F_{t} X_{t}\right] .
$$

Remark. Note that before being proved in its general form in [10], Theorem 1.1 was shown (under the usual assumptions) by Cheridito, Nikeghbali and Platen [6] in the particular case where the submartingale $X$ is of class $(D)$ (in fact, as mentioned in [6], the solution is essentially contained, somewhat hidden, in [1]). In this case, the measure $\mathcal{Q}$ is finite and satisfies

$$
\mathcal{Q}=X_{\infty} \mathbb{P}
$$

Moreover, Theorem 1.1 has already been obtained in some special cases such as the case where $X_{t}$ is the absolute value of the canonical process on the Wiener 
space or when $X_{t}=\left|Y_{t}\right|^{\alpha-1}$ where $Y$ is a symmetric stable Lévy process of index $\alpha \in(1,2)$, although in this latter case the property (1.3) was not noticed ([16]). In fact, almost all our results will apply to a large class of symmetric Lévy processes including the symmetric stable Lévy processes of index $\alpha \in(1,2)$. We shall now detail this last example a little more since it provides natural examples of processes with jumps, living on the Skorokhod space. Let us define, on the space $\mathcal{D}\left(\mathbb{R}_{+}, \mathbb{R}\right)$, $\left(\mathcal{F}_{t}\right)_{t \geq 0}$ as the natural filtration of the canonical process $\left(Y_{t}\right)_{t \geq 0}$, and let us set

$$
\mathcal{F}:=\bigvee_{t \geq 0} \mathcal{F}_{t}
$$

We consider on $\mathcal{D}\left(\mathbb{R}_{+}, \mathbb{R}\right)$ the probability $\mathbb{P}$ under which $\left(Y_{t}\right)_{t \geq 0}$ is a symmetric Lévy process starting from zero, with exponent $\Psi$ :

$$
\mathbb{P}\left[\exp \left(i \xi Y_{t}\right)\right]=\exp (-t \Psi(\xi)) .
$$

Moreover, we assume that 0 is regular for itself and that $\left(Y_{t}\right)_{t \geq 0}$ is recurrent, or equivalently (see Bertoin [4]),

$$
\int_{-\infty}^{\infty} \frac{d \xi}{1+\Psi(\xi)}<\infty \quad \text { and } \quad \int_{0} \frac{d \xi}{\Psi(\xi)}=\infty
$$

In the case where $\left(Y_{t}\right)_{t \geq 0}$ is a symmetric $\alpha$-stable Lévy process of index $\alpha \in(1,2)$, we have $\Psi(\xi)=|\xi|^{\alpha}$ and the above conditions on $\Psi$ are satisfied (see [4]). Salminen and Yor [15] have proved that if for some $x \in \mathbb{R}$,

$$
v(x)=\frac{1}{\pi} \int_{0}^{\infty} \frac{1-\cos (\xi x)}{\Psi(\xi)} d \xi<\infty,
$$

then

$$
v\left(Y_{t}-x\right)=v(x)+N_{t}^{x}+L_{t}^{x},
$$

where $N_{t}^{x}$ is a martingale and where $\left(L_{t}^{x}\right)_{t \geq 0}$ is the local time at level $x$ of the Lévy process $\left(Y_{t}\right)_{t \geq 0}$. Since $\left(L_{t}^{x}\right)_{t \geq 0}$ is continuous, increasing, adapted and only increases when $Y_{t}=x$ (see Bertoin [4, Chapter V]), the process $\left(v\left(Y_{t}-x\right)\right)_{t \geq 0}$ is of class $(\Sigma)$, moreover, $\left(Y_{t}\right)_{t \geq 0}$ is recurrent and 0 is regular for itself, which implies that $\lim _{t \rightarrow \infty} L_{t}^{x}=\infty \mathbb{P}$-almost surely. Hence Theorem 1.1 applies, and for any $x \in \mathbb{R}$, there exists a $\sigma$-finite measure $\mathcal{Q}_{x}$, singular to $\mathbb{P}$ and such that all the properties of Theorem 1.1 are satisfied with $X_{t}=v\left(Y_{t}-x\right)$ and $g \equiv g_{x}=$ $\sup \left\{t: Y_{t}=x\right\}$. In the special case of symmetric $\alpha$-stable Lévy processes of index $\alpha \in(1,2), v(x)=c(\alpha)|x|^{\alpha-1}$ for some explicit constant $c(\alpha)$ (see [15]). In what follows, all our results which do not require any assumptions on the sign of the jumps will apply to this family of examples as well. 
Let us now briefly recall the general construction of $\mathcal{Q}$ (defined in Theorem 1.1) given in [10]. For a Borel, integrable, strictly positive and bounded function $f$ from $\mathbb{R}$ to $\mathbb{R}$, one defines a function $G$ as

$$
G(x)=\int_{x}^{\infty} f(y) d y,
$$

and then one proves that the process

$$
\left(M_{t}^{f}:=G\left(A_{t}\right)-\mathbb{P}\left[G\left(A_{\infty}\right) \mid \mathcal{F}_{t}\right]+f\left(A_{t}\right) X_{t}\right)_{t \geq 0}
$$

is a martingale with respect to $\mathbb{P}$ and the filtration $\left(\mathcal{F}_{t}\right)_{t \geq 0}$. Since $\left(\Omega, \mathcal{F},\left(\mathcal{F}_{t}\right)_{t \geq 0}, \mathbb{P}\right)$ satisfies the natural conditions and $G\left(A_{t}\right) \geq G\left(A_{\infty}\right)$, one can suppose that this martingale is nonnegative and càdlàg, by choosing the version of $\mathbb{P}\left[G\left(A_{\infty}\right) \mid \mathcal{F}_{t}\right]$ carefully. In this case, since $\left(\Omega, \mathcal{F},\left(\mathcal{F}_{t}\right)_{t \geq 0}, \mathbb{P}\right)$ has property $(\mathrm{NP})$, there exists a unique finite measure $\mathcal{M}^{f}$ such that for all $t \geq 0$, and for all bounded, $\mathcal{F}_{t^{-}}$ measurable functionals $\Gamma_{t}$,

$$
\mathcal{M}^{f}\left[\Gamma_{t}\right]=\mathbb{P}\left[\Gamma_{t} M_{t}^{f}\right] .
$$

Now, since $f$ is strictly positive, one can define a $\sigma$-finite measure $\mathcal{Q}^{f}$ by,

$$
\mathcal{Q}^{f}:=\frac{1}{f\left(A_{\infty}\right)} \mathcal{M}^{f} .
$$

It is proved in [10] that if $G / f$ is unformly bounded (this condition is, for example, satisfied for $\left.f(x)=e^{-x}\right)$, then $\mathcal{Q}^{f}$ satisfies the conditions defining $\mathcal{Q}$ in Theorem 1.1, which implies the existence part of that result. The uniqueness part is then proved in a very easy way; one remarkable consequence of the uniqueness is the fact that $\mathcal{Q}^{f}$ does not depend on the choice of $f$.

One of the remarkable features of the measure $\mathcal{Q}$, in the special case of the Wiener space and when $\left(X_{t}\right)_{t \geq 0}$ is the absolute value of the Wiener process, is that it allows a unified view of some penalization problems related to Wiener measure. More precisely, Roynette, Vallois and Yor [14] consider $\mathbb{W}$, the Wiener measure on the space $\mathcal{C}\left(\mathbb{R}_{+}, \mathbb{R}\right)$ endowed with its canonical filtration $\left(\mathcal{F}_{s}\right)_{s \geq 0}$ (not completed), and then they define $\mathcal{F}:=\bigvee_{s \geq 0} \mathcal{F}_{s}$. They consider a family $\left(\Gamma_{t}\right)_{t \geq 0}$ of nonnegative random variables on the same space such that

$$
0<\mathbb{W}\left[\Gamma_{t}\right]<\infty
$$

and for $t \geq 0$, they define the probability measure

$$
\mathbb{Q}_{t}:=\frac{\Gamma_{t}}{\mathbb{W}\left[\Gamma_{t}\right]} \mathbb{W} .
$$

Then they are able to prove that for many examples of families of functionals 
$\left(\Gamma_{t}\right)_{t>0}$, there exists a probability measure $\mathbb{Q}_{\infty}$ which can be considered as the weak limit of $\left(\mathbb{Q}_{t}\right)_{t \geq 0}$ as $t \rightarrow \infty$, in the following sense: for all $s \geq 0$ and for all events $\Lambda_{s} \in \mathcal{F}_{s}$

$$
\mathbb{Q}_{t}\left[\Lambda_{s}\right] \underset{t \rightarrow \infty}{\longrightarrow} \mathbb{Q}_{\infty}\left[\Lambda_{s}\right] .
$$

Finding the measure $\mathbb{Q}_{\infty}$ amounts to solving the penalization problem associated with the functionals $\left(\Gamma_{t}\right)_{t \geq 0}$. The functional $\Gamma_{t}$ is typically some function of the local time or the running supremum of the Wiener process, or some Feynman-Kac functional of the Wiener process. In the monograph [11], Najnudel, Roynette and Yor have proved that the measure $\mathcal{Q}$ associated with the absolute value of the Wiener process allows a unified approach to many examples dealt with separately in the literature; under some technical conditions on the functionals $\left(\Gamma_{t}\right)_{t \geq 0}$, they show that the measure $\mathbb{Q}_{\infty}$ is absolutely continuous with respect to $\mathcal{Q}$ with an explicit density. In this paper, we shall completely solve the penalization problem (under the assumptions of Theorem 1.1) with functionals of the form $\Gamma_{t}=F_{t} X_{t}$, where $F_{t}$ is some functional satisfying a not too restrictive condition. In particular we need no assumption on the continuity of the paths of $\left(X_{t}\right)$, nor any Markov or scaling properties.

More precisely, throughout this paper, we establish some of the fundamental properties of the measure $\mathcal{Q}$ (which also prepare the ground for a forthcoming work on penalization of diffusion paths). A remarkable class of martingales defined as local densities (with respect to $\mathbb{P}$ ) of finite measures, absolutely continuous with respect to $\mathcal{Q}$, is involved in a crucial way. The precise definition of these martingales is given in Section 2, and they are explicitly computed in some particular cases. In Section 3 , we study their behaviour as $t \rightarrow \infty$, in the most interesting case where $A_{\infty}=\infty \mathbb{P}$-almost surely, and we deduce some information about the behaviour of $\left(X_{t}\right)_{t \geq 0}$ under the measure $\mathcal{Q}$. We then naturally deduce the announced universal penalization results from our study of the asymptotic behaviour of these martingales and of $\left(X_{t}\right)_{t \geq 0}$ under $\mathcal{Q}$. In Section 4 , we give a decomposition of any nonnegative supermartingale which can be interpreted as the decomposition of a finite measure on $(\Omega, \mathcal{F})$ as the sum of three measures, one of them being absolutely continuous with respect to $\mathbb{P}$, the second absolutely continuous with respect to $\mathcal{Q}$, and the last one singular with respect to $\mathbb{P}$ and $\mathcal{Q}$.

\section{§2. A remarkable class of martingales related to the measures $\mathcal{Q}$}

We assume that we are working under the assumptions of Definition 1.1 and Theorem 1.1. Let us first remark that since $\mathcal{Q}[g=\infty]=0$, one has

$$
\mathcal{Q}\left[A_{\infty}=\infty\right]=0,
$$


i.e. $A_{\infty}$ is finite $\mathcal{Q}$-almost everywhere. Let us state a useful result which was proved in [10]:

Proposition 2.1. Let $f$ be an integrable function from $\mathbb{R}_{+}$to $\mathbb{R}_{+}$. Then under the assumptions of Theorem 1.1, the measure

$$
\mathcal{M}^{f}:=f\left(A_{\infty}\right) \mathcal{Q}
$$

is the unique positive, finite measure such that for all $t \geq 0$, and for all bounded, $\mathcal{F}_{t}$-measurable functionals $\Gamma_{t}$,

$$
\mathcal{M}^{f}\left[\Gamma_{t}\right]=\mathbb{P}\left[\Gamma_{t} M_{t}^{f}\right]
$$

where the process $\left(M_{t}^{f}\right)_{t \geq 0}$ is given by

$$
M_{t}^{f}:=G\left(A_{t}\right)-\mathbb{P}\left[G\left(A_{\infty}\right) \mid \mathcal{F}_{t}\right]+f\left(A_{t}\right) X_{t} \quad \text { for } \quad G(x):=\int_{x}^{\infty} f(y) d y .
$$

In particular, $\left(M_{t}^{f}\right)_{t \geq 0}$ is a martingale, càdlàg if one chooses a suitable version of the conditional expectation of $G\left(A_{\infty}\right)$ given $\mathcal{F}_{t}$. Moreover, $\left(M_{t}^{f}\right)_{t \geq 0}$ is uniquely determined by $f$ in the following sense: two càdlàg martingales satisfying (2.2) are necessarily indistinguishable.

Proposition 2.1 gives a relation between a finite measure which is absolutely continuous with respect to $\mathcal{Q}\left(\mathcal{M}^{f}\right)$, and a càdlàg martingale $\left(M_{t}^{f}\right)_{t \geq 0}$. This relation can be generalized as follows:

Theorem 2.1. Under the assumptions and notation of Theorem 1.1, let $F$ be a $\mathcal{Q}$ integrable, nonnegative functional defined on $(\Omega, \mathcal{F})$. Define also the finite measure $\mathcal{M}^{F}:=F \mathcal{Q}$. Then there exists a càdlàg $\mathbb{P}$-martingale $\left(M_{t}(F)\right)_{t \geq 0}$ such that for all $t \geq 0$, and for all bounded, $\mathcal{F}_{t}$-measurable functionals $\Gamma_{t}$,

$$
\mathcal{M}^{F}\left[\Gamma_{t}\right]=\mathbb{P}\left[\Gamma_{t} M_{t}(F)\right] .
$$

The martingale $\left(M_{t}(F)\right)_{t \geq 0}$ is unique up to indistinguishability.

Proof. Let $t \geq 0$, let $\Gamma_{t}$ be a nonnegative, $\mathcal{F}_{t}$-measurable functional such that

$$
\mathbb{P}\left[\Gamma_{t}\right]=0,
$$

and let $f$ be an integrable, strictly positive function from $\mathbb{R}_{+}$to $\mathbb{R}_{+}$. Then

$$
\mathbb{P}\left[M_{t}^{f} \Gamma_{t}\right]=0,
$$

and by Proposition 2.1,

$$
\mathcal{Q}\left[f\left(A_{\infty}\right) \Gamma_{t}\right]=0
$$


Since $f$ is supposed to be strictly positive, one deduces that $\mathcal{Q}\left[\Gamma_{t}\right]=0$, and finally $\mathcal{Q}\left[F \Gamma_{t}\right]=0$. Therefore, the restriction of the finite measure $\mathcal{M}^{F}$ to $\mathcal{F}_{t}$ is absolutely continuous with respect to $\mathbb{P}$, and there exists a nonnegative, $\mathcal{F}_{t}$-measurable random variable $M_{t}^{(0)}$ such that for all $\mathcal{F}_{t}$-measurable, bounded variables $\Gamma_{t}$,

$$
\mathcal{M}^{F}\left[\Gamma_{t}\right]=\mathbb{P}\left[M_{t}^{(0)} \Gamma_{t}\right]
$$

This equality, holding for all $t \geq 0$, implies that $\left(M_{t}^{(0)}\right)_{t \geq 0}$ is a $\mathbb{P}$-martingale. Since the underlying probability space satisfies the natural conditions, $\left(M_{t}^{(0)}\right)_{t \geq 0}$ admits a càdlàg modification $\left(M_{t}\right)_{t \geq 0}$, and

$$
\mathcal{M}^{F}\left[\Gamma_{t}\right]=\mathbb{P}\left[M_{t} \Gamma_{t}\right] .
$$

By the monotone class theorem this determines the measure $\mathcal{M}^{F}$ uniquely. Moreover, if $\left(M_{t}^{\prime}\right)_{t \geq 0}$ is a càdlàg martingale satisfying

$$
\mathcal{M}^{F}\left[\Gamma_{t}\right]=\mathbb{P}\left[M_{t}^{\prime} \Gamma_{t}\right],
$$

then for all $t \geq 0, M_{t}=M_{t}^{\prime}$ almost surely, and since $M$ and $M^{\prime}$ are càdlàg, they are indistinguishable.

By Theorem 2.1, one can define a particular family of nonnegative, càdlàg $\mathbb{P}$ martingales: those of the form $\left(M_{t}(F)\right)_{t \geq 0}$, where $F$ is a nonnegative, $\mathcal{Q}$-integrable functional $F$. By construction, they correspond to the local densities, with respect to $\mathbb{P}$, of finite measures which are absolutely continuous with respect to $\mathcal{Q}$. The situation is similar to the case of nonnegative, uniformly integrable martingales, which are the local densities of finite measures, absolutely continuous with respect to $\mathbb{P}$. Theorem 2.1 does not give any explicit formula for the martingale $M_{t}(F)$. However, from Proposition 2.1, one immediately deduces the following result:

Corollary 2.1. Under the assumptions of Theorem 1.1, for all integrable functions $f$ from $\mathbb{R}_{+}$to $\mathbb{R}_{+}, f\left(A_{\infty}\right)$ is integrable with respect to $\mathcal{Q}$, and the martingale $\left(M_{t}\left(f\left(A_{\infty}\right)\right)\right)_{t \geq 0}$ is indistinguishable from the martingale $\left(M_{t}^{f}\right)_{t \geq 0}$ defined in Proposition 2.1 .

Remark. Let $f$ be an integrable function from $\mathbb{R}_{+}$to $\mathbb{R}_{+}$. The martingale

$$
\left(\mathbb{P}\left[G\left(A_{\infty}\right) \mid \mathcal{F}_{t}\right]\right)_{t \geq 0}
$$

admits a càdlàg version. If it is denoted by $\left(G_{t}\right)_{t \geq 0}$, one has

$$
M_{t}(F)=G\left(A_{t}\right)-G_{t}+Y_{t},
$$

where $\left(Y_{t}\right)_{t \geq 0}$ is a càdlàg modification of $\left(f\left(A_{t}\right) X_{t}\right)_{t \geq 0}$, which then exists for any choice of $f$ (recall that $G\left(A_{t}\right)$ is continuous in $t$ ). If $f$ is bounded, one easily proves 
that $f\left(A_{t}\right) X_{t}$ is càdlàg with respect to $t$; in this case, $\left(Y_{t}\right)_{t \geq 0}$ is indistinguishable from $\left(f\left(A_{t}\right) X_{t}\right)_{t \geq 0}$. However, for unbounded $f$, the existence of $Y$ is not trivial.

Another case for which one can give a simple expression for the martingale $\left(M_{t}(F)\right)_{t \geq 0}$ is when $\left(X_{t}\right)_{t \geq 0}$ is of class (D). More precisely, one has the following result:

Theorem 2.2. Suppose that the assumptions of Theorem 1.1 are satisfied, and that the process $\left(X_{t}\right)_{t \geq 0}$ is of class (D). Then $X_{t}$ tends a.s. to a limit $X_{\infty}$ as $t \rightarrow \infty$, and the measure $\mathcal{Q}$ is absolutely continuous with respect to $\mathbb{P}$, with density $X_{\infty}$. Moreover, a nonnegative measurable functional $F$ is integrable with respect to $\mathcal{Q}$ if and only if $F X_{\infty}$ is integrable with respect to $\mathbb{P}$, and in this case, $\left(M_{t}(F)\right)_{t \geq 0}$ is a càdlàg version (unique up to indistinguishability) of the conditional expectation $\left(\mathbb{P}\left[F X_{\infty} \mid \mathcal{F}_{t}\right]\right)_{t \geq 0}$. In particular, it is uniformly integrable, and it converges a.s. and in $L^{1}$ to $F X_{\infty}$ as $t \rightarrow \infty$.

Proof. The equality $\mathcal{Q}=X_{\infty} \mathbb{P}$ is contained in [10], [1] and [6]. Let us briefly reprove it here. Since $\left(X_{t}\right)_{t>0}$ is of class (D), the expectation of $A_{t}$ is bounded, and so $A_{\infty}$ is integrable, which implies that $\left(N_{t}\right)_{t>0}$ is a uniformly integrable, càdlàg martingale. It admits an a.s. limit $N_{\infty}$ as $t \rightarrow \infty$, and hence $X_{\infty}$ is welldefined. Moreover, if $d_{t}$ is the infimum of $u>t$ such that $X_{u}=0$, by the version of the début theorem given in [8], $d_{t}$ is a stopping time. Moreover, $d_{t}=\infty$ if and only if $g \leq t$, and by right-continuity of $X, X_{d_{t}}=0$ for $d_{t}<\infty$. One deduces

$$
\mathbb{P}\left[X_{\infty} \mathbb{1}_{g \leq t} \mid \mathcal{F}_{t}\right]=\mathbb{P}\left[X_{\infty} \mathbb{1}_{d_{t}=\infty} \mid \mathcal{F}_{t}\right]=\mathbb{P}\left[X_{d_{t}} \mid \mathcal{F}_{t}\right]
$$

Now, since $\left(N_{t}\right)_{t \geq 0}$ is a uniformly integrable, càdlàg martingale,

$$
\mathbb{P}\left[X_{\infty} \mathbb{1}_{g \leq t} \mid \mathcal{F}_{t}\right]=\mathbb{P}\left[N_{d_{t}}+A_{d_{t}} \mid \mathcal{F}_{t}\right]=N_{t}+A_{t}=X_{t}
$$

(we have used above the fact that $A_{d_{t}}=A_{t}$ : since $\left(X_{t}\right)_{t \geq 0}$ is of class $(\Sigma)$, the process $u \rightarrow A_{u}$ is constant for $t \leq u \leq d_{t}$ ), or equivalently, for all bounded, $\mathcal{F}_{t}$-measurable functionals $\Gamma_{t}$,

$$
\mathbb{P}\left[\Gamma_{t} X_{\infty} \mathbb{1}_{g \leq t}\right]=\mathbb{P}\left[\Gamma_{t} X_{t}\right]
$$

Moreover, under $X_{\infty} \mathbb{P}, X_{\infty}>0$ almost everywhere and so $g<\infty$. One deduces that $X_{\infty} \mathbb{P}$ is equal to $\mathcal{Q}$. For any nonnegative functional $F$, it is then trivial that $F$ is integrable with respect to $\mathcal{Q}$ if and only if $F X_{\infty}$ is integrable with respect to $\mathbb{P}$; in this case, the finite measure $\mathcal{M}^{F}$ has density $F X_{\infty}$ with respect to $\mathbb{P}$. By taking the restriction to $\mathcal{F}_{t}$, one deduces that the martingale $\left(M_{t}(F)\right)_{t \geq 0}$ is a càdlàg version of the conditional expectation of $F X_{\infty}$. 
It is also possible to describe $\mathcal{Q}$ and $\left(M_{t}(F)\right)_{t \geq 0}$ explicitly if $\left(X_{t}\right)_{t \geq 0}$ is a strictly positive martingale:

Theorem 2.3. Suppose that the assumptions of Theorem 1.1 are satisfied, and that $\mathbb{P}$-almost surely, $\left(X_{t}\right)_{t \geq 0}$ does not vanish; in particular, $\left(A_{t}\right)_{t \geq 0}$ is indistinguishable from zero, and $\left(X_{t}\right)_{t \geq 0}$ is a martingale. Then $\mathcal{Q}$ is finite and it is the unique measure such that for $t \geq 0$, the restriction of $\mathcal{Q}$ to $\mathcal{F}_{t}$ has density $X_{t}$ with respect to the restriction of $\mathbb{P}$ to $\mathcal{F}_{t}$. Moreover, for any nonnegative, $\mathcal{Q}$-integrable functional $F$, the martingale $\left(M_{t}(F)\right)_{t \geq 0}$ can be given by

$$
M_{t}(F)=X_{t} \widetilde{\mathcal{Q}}\left[F \mid \mathcal{F}_{t}\right]
$$

where $\widetilde{\mathcal{Q}}\left[F \mid \mathcal{F}_{t}\right]$ is a càdlàg version of the conditional expectation of $F$ given $\mathcal{F}_{t}$, with respect to the probability measure $\widetilde{\mathcal{Q}}$ obtained by dividing $\mathcal{Q}$ by its total mass (different from zero). In particular, the functional identically equal to one is $\mathcal{Q}$ integrable and $\left(M_{t}(1)\right)_{t \geq 0}$ is indistinguishable from $\left(X_{t}\right)_{t \geq 0}$.

Proof. Let $T_{0}:=\inf \left\{t \geq 0: X_{t}=0\right\}$. By the début theorem (under the natural conditions), $T_{0}$ is an $\left(\mathcal{F}_{t}\right)_{t \geq 0}$-stopping time. By assumption, for all $t \geq 0$, the event $\left\{T_{0}>t\right\}$, which is in $\mathcal{F}_{t}$, holds $\mathbb{P}$-almost surely. Now, by the construction of $\mathcal{Q}$ given in [10] and described above, $\mathcal{Q}$ is absolutely continuous with respect to a finite measure which is locally absolutely continuous with respect to $\mathbb{P}$. One deduces that for all $t \geq 0$, the event $\left\{T_{0}>t\right\}$ holds $\mathcal{Q}$-almost everywhere. Hence, $\mathcal{Q}$-almost everywhere, $T_{0}$ is infinite and $\left(X_{t}\right)_{t \geq 0}$ does not vanish, which implies

$$
\mathcal{Q}\left[\Gamma_{t}\right]=\mathbb{P}\left[\Gamma_{t} X_{t}\right] .
$$

By the monotone class theorem, $\mathcal{Q}$ is the unique measure satisfying (2.3); it is finite since $X_{0}$ is integrable, its total mass is different from zero since $X_{0}>0$. Hence, $\widetilde{\mathcal{Q}}$ is well-defined. Moreover, if $F$ is integrable with respect to $\mathcal{Q}$, it is also integrable with respect to $\widetilde{\mathcal{Q}}$, and the $\widetilde{\mathcal{Q}}$-martingale

$$
\left(\widetilde{\mathcal{Q}}\left[F \mid \mathcal{F}_{t}\right]\right)_{t \geq 0}
$$

is well-defined and admits a càdlàg version $\left(Y_{t}\right)_{t \geq 0}$. Indeed, $\left(\Omega, \mathcal{F},\left(\mathcal{F}_{t}\right)_{t \geq 0}, \mathbb{P}\right)$ satisfies the natural conditions, and hence so does $\left(\Omega, \mathcal{F},\left(\mathcal{F}_{t}\right)_{t \geq 0}, \widetilde{\mathcal{Q}}\right)$, since for all $t \geq 0$, the restriction of $\widetilde{\mathcal{Q}}$ to $\mathcal{F}_{t}$ is equivalent to the restriction of $\mathbb{P}$ (recall that $X_{t}>0 \mathbb{P}$-almost surely). Therefore, for all bounded, $\mathcal{F}_{t}$-measurable functionals $\Gamma_{t}$,

$$
\mathcal{Q}\left[F \Gamma_{t}\right]=\mathcal{Q}[1] \widetilde{\mathcal{Q}}\left[F \Gamma_{t}\right]=\mathcal{Q}[1] \widetilde{\mathcal{Q}}\left[\Gamma_{t} \widetilde{\mathcal{Q}}\left[F \mid \mathcal{F}_{t}\right]\right]=\mathcal{Q}\left[\Gamma_{t} Y_{t}\right]=\mathbb{P}\left[\Gamma_{t} X_{t} Y_{t}\right]
$$

Now,

$$
\mathcal{Q}\left[F \Gamma_{t}\right]=\mathcal{M}^{F}\left[\Gamma_{t}\right]=\mathbb{P}\left[\Gamma_{t} M_{t}(F)\right]
$$


with the notation of Theorem 2.1. Hence, $\left(M_{t}(F)\right)_{t \geq 0}$ is a modification of $\left(X_{t} Y_{t}\right)_{t \geq 0}$, and since these two processes are càdlàg, they are indistinguishable. Moreover, the functional equal to one is $\mathcal{Q}$-integrable, since $\mathcal{Q}$ is finite. In this case, one can take $Y_{t}=1$ for all $t \geq 0$, and $\left(M_{t}(F)\right)_{t \geq 0}$ is indistinguishable from $\left(X_{t}\right)_{t \geq 0}$.

After giving these simple examples for which one can explicitly compute $\mathcal{Q}$ and $M_{t}(F)$, it is natural to ask what happens in a more general situation. In Section 3 , we study the case where $A_{\infty}=\infty \mathbb{P}$-almost surely (this case occurs, in particular, when $\left(X_{t}\right)_{t \geq 0}$ is a reflected Brownian motion). Unfortunately, we are not able to give explicit expressions for the martingales of the form $\left(M_{t}(F)\right)_{t>0}$ in this case, but we obtain some information about their behaviour as $t \rightarrow \infty$.

\section{§3. The case $A_{\infty}=\infty$ under $\mathbb{P}$}

In this case, let us first recall the following result, proven in [10]:

Proposition 3.1. Suppose that the assumptions of Theorem 1.1 are satisfied, and that $A_{\infty}=\infty \mathbb{P}$-almost surely. Then the image of $\mathcal{Q}$ under the functional $A_{\infty}$ is the measure

$$
\mathbb{P}\left[X_{0}\right] \delta_{0}+\lambda,
$$

where $\lambda$ is Lebesgue measure on $\mathbb{R}_{+}$. In particular $\mathcal{Q}$ is an infinite measure.

Moreover, again for $A_{\infty}=\infty$, the martingale $\left(M_{t}(F)\right)_{t \geq 0}$ tends $\mathbb{P}$-almost surely to zero for any $\mathcal{Q}$-integrable functional $F$. In particular, it cannot be uniformly integrable, except for $F=0 \mathcal{Q}$-almost everywhere. More precisely, one has the slightly more general result:

Proposition 3.2. Suppose the assumptions of Theorem 1.1 hold. Then on the set $\left\{A_{\infty}=\infty\right\}$, for every $\mathcal{Q}$-integrable, nonnegative functional $F$ defined on $(\Omega, \mathcal{F})$, the martingale $M_{t}(F)$ tends $\mathbb{P}$-almost surely to zero as $t \rightarrow \infty$.

Proof. Let us use the notation of Theorem 2.1. For all $u>0$ and $v \geq t>0$,

$$
\mathcal{M}^{F}\left[A_{t}>u\right]=\mathbb{P}\left[M_{v}(F) \mathbb{1}_{A_{t}>u}\right] .
$$

Moreover, $\mathbb{P}$-almost surely

$$
M_{v}(F) \mathbb{1}_{A_{t}>u} \underset{v \rightarrow \infty}{\longrightarrow} M_{\infty}(F) \mathbb{1}_{A_{t}>u}
$$

where $M_{\infty}(F)$ is the a.s. limit of $M_{t}(F)$ as $t \rightarrow \infty$ (recall that $M_{t}(F)$ is a nonnegative martingale under $\mathbb{P}$ and hence converges almost surely). By Fatou's lemma, 
one deduces

$$
\mathbb{P}\left[M_{\infty}(F) \mathbb{1}_{A_{t}>u}\right] \leq \mathcal{M}^{F}\left[A_{t}>u\right] \leq \mathcal{M}^{F}\left[A_{\infty}>u\right] .
$$

Now, $M_{\infty}(F) \mathbb{1}_{A_{\infty}>u}$ is the almost sure limit of $M_{\infty}(F) \mathbb{1}_{A_{t}>u}$ as $t \rightarrow \infty$. Since $M_{\infty}(F)$ is integrable with respect to $\mathbb{P}$ by Fatou's lemma, one has, by dominated convergence,

$$
\mathbb{P}\left[M_{\infty}(F) \mathbb{1}_{A_{\infty}>u}\right] \leq \mathcal{M}^{F}\left[A_{\infty}>u\right] .
$$

By letting $t \rightarrow \infty$, we are done, since $A_{\infty}$ is finite $\mathcal{M}^{F}$-almost everywhere (this is a consequence of (2.1) and the fact that $\mathcal{M}^{F}$ is absolutely continuous with respect to $\mathcal{Q})$.

Once the behaviour of $\left(M_{t}(F)\right)_{t \geq 0}$ under $\mathbb{P}$ is known, it is natural to ask what happens under $\mathcal{Q}$. The following result implies that the behaviour of $\left(M_{t}(F)\right)_{t \geq 0}$ is not the same as under $\mathbb{P}$. Moreover, it gives some information about the behaviour of $\left(X_{t}\right)_{t \geq 0}$ under $\mathcal{Q}$ :

Theorem 3.1. Suppose that the assumptions of Theorem 1.1 are satisfied, and that $A_{\infty}=\infty \mathbb{P}$-almost surely. Then $\mathcal{Q}$-almost everywhere, $X_{t}$ tends to infinity with $t$, and

$$
\frac{M_{t}(F)}{X_{t}} \underset{t \rightarrow \infty}{\longrightarrow} F
$$

for all nonnegative, $\mathcal{Q}$-integrable functionals $F$.

Remark. As we have seen in Theorem 2.1, two càdlàg versions of $\left(M_{t}(F)\right)_{t \geq 0}$ are indistinguishable with respect to $\mathbb{P}$. Since $\mathcal{Q}$ is absolutely continuous with respect to a finite measure which is locally absolutely continuous with respect to $\mathbb{P}$, the two versions are also indistinguishable with respect to $\mathcal{Q}$. Hence, $\left(M_{t}(F)\right)_{t \geq 0}$ can be considered to be well-defined for all problems concerning its behaviour under the measure $\mathcal{Q}$.

Remark. In the case where $\left(X_{t}\right)_{t \geq 0}$ is a reflected Brownian motion, Theorem 3.1 is essentially proved in [11] and when $X_{t}$ is $\left|Y_{t}\right|^{\alpha-1}$ for a symmetric $\alpha$-stable process $\left(Y_{t}\right)_{t \geq 0}$ of index $\alpha \in(1,2)$, it is proved in [16].

Proof of Theorem 3.1. The functional $H:=e^{-A_{\infty}}$ is $\mathcal{Q}$-integrable and one has (from Corollary 2.1)

$$
M_{t}(H)=e^{-A_{t}}\left(1+X_{t}\right)
$$

(recall that $\mathbb{P}$-almost surely, $e^{-A_{\infty}}=0$, since $A_{\infty}=\infty$ ). One deduces that, for all bounded, $\mathcal{F}_{t}$-measurable random variables $\Gamma_{t}$

$$
\mathcal{M}^{H}\left[\Gamma_{t}\right]=\mathbb{P}\left[e^{-A_{t}}\left(1+X_{t}\right) \Gamma_{t}\right]
$$


This implies

$$
\mathbb{P}\left[\Gamma_{t}\right]=\mathcal{M}^{H}\left[Y_{t} \Gamma_{t}\right], \quad \text { where } \quad Y_{t}=\frac{e^{A_{t}}}{1+X_{t}} .
$$

Now, $\left(Y_{t}\right)_{t \geq 0}$ is a nonnegative, càdlàg martingale with respect to the probability measure $\widetilde{\mathcal{M}}^{H}:=\mathcal{M}^{H} / \mathcal{M}^{H}(1)$, and so it converges $\mathcal{M}^{H}$-almost everywhere to a limit random variable $Y_{\infty}$. Now, since for all $u>0$ and $v \geq t>0$,

$$
\mathbb{P}\left[A_{t} \leq u\right]=\mathcal{M}^{H}\left[Y_{v} \mathbb{1}_{A_{t} \leq u}\right],
$$

one has, by letting $v \rightarrow \infty$ and by using Fatou's lemma,

$$
\mathcal{M}^{H}\left[Y_{\infty} \mathbb{1}_{A_{t} \leq u}\right] \leq \mathbb{P}\left[A_{t} \leq u\right],
$$

which implies

$$
\mathcal{M}^{H}\left[Y_{\infty} \mathbb{1}_{A_{\infty} \leq u}\right] \leq \mathbb{P}\left[A_{t} \leq u\right] .
$$

Now, since $A_{\infty}=\infty \mathbb{P}$-almost surely, one has

$$
\mathbb{P}\left[A_{t} \leq u\right] \underset{t \rightarrow \infty}{\longrightarrow} 0 .
$$

Hence,

$$
\mathcal{M}^{H}\left[Y_{\infty} \mathbb{1}_{A_{\infty} \leq u}\right]=0,
$$

and finally (by letting $u \rightarrow \infty$ )

$$
\mathcal{M}^{H}\left[Y_{\infty} \mathbb{1}_{A_{\infty}<\infty}\right]=0 .
$$

Since $A_{\infty}<\infty \mathcal{Q}$-almost everywhere, $Y_{\infty}=0 \mathcal{M}^{H}$-almost everywhere, which implies that $X_{t}$ tends to infinity with $t$. On the other hand, for all nonnegative, integrable functionals $F$, and for all bounded, $\mathcal{F}_{t}$-measurable functionals $\Gamma_{t}$, one has

$$
\begin{aligned}
\mathcal{M}^{H}\left[\Gamma_{t} \frac{M_{t}(F)}{M_{t}(H)}\right] & =\mathcal{Q}\left[\Gamma_{t} H \frac{M_{t}(F)}{M_{t}(H)}\right]=\mathbb{P}\left[\Gamma_{t} M_{t}(H) \frac{M_{t}(F)}{M_{t}(H)}\right]=\mathbb{P}\left[\Gamma_{t} M_{t}(F)\right] \\
& =\mathcal{Q}\left[\Gamma_{t} F\right]=\mathcal{M}^{H}\left[\Gamma_{t} \frac{F}{H}\right]=\mathcal{M}^{H}\left[\Gamma_{t} \widetilde{\mathcal{M}}^{H}\left[\frac{F}{H} \mid \mathcal{F}_{t}\right]\right] .
\end{aligned}
$$

Note that all the equalities above are meaningful since $M_{t}(H)$ and $H$ never vanish. Therefore, for all $t \geq 0$, one has almost surely

$$
\frac{M_{t}(F)}{M_{t}(H)}=\widetilde{\mathcal{M}}^{H}\left[\frac{F}{H} \mid \mathcal{F}_{t}\right],
$$

which implies that

$$
\frac{M_{t}(F)}{M_{t}(H)} \underset{t \rightarrow \infty}{\longrightarrow} \frac{F}{H}
$$


$\widetilde{\mathcal{M}}^{H}$-almost surely, and hence $\mathcal{Q}$-almost everywhere. Now, since $X_{t} \rightarrow \infty \mathcal{Q}$-almost everywhere, $X_{t}>0$ for $t$ large enough and

$$
\frac{M_{t}(H)}{X_{t}}=e^{-A_{t}}\left(1+\frac{1}{X_{t}}\right) \underset{t \rightarrow \infty}{\longrightarrow} e^{-A_{\infty}}
$$

One deduces that

$$
\frac{M_{t}(F)}{X_{t}}=\frac{M_{t}(F)}{M_{t}(H)} \frac{M_{t}(H)}{X_{t}} \underset{t \rightarrow \infty}{\longrightarrow} \frac{F}{H} e^{-A_{\infty}}=F .
$$

In the particular case of the reflected Brownian motion, the measure $\mathcal{Q}$ is strongly related to the last passage time at any level and not only at zero. This relation can be generalized as follows:

Theorem 3.2. Suppose that the assumptions of Theorem 1.1 are satisfied, that the submartingale $\left(X_{t}\right)_{t \geq 0}$ has only positive jumps and that $A_{\infty}=\infty$ almost surely under $\mathbb{P}$. For $a \geq 0$, let $g^{[a]}$ be the last hitting time of the interval $[0, a]$,

$$
g^{[a]}=\sup \left\{t \geq 0: X_{t} \leq a\right\}
$$

Then for all $t \geq 0$, and for all $\mathcal{F}_{t}$-measurable, bounded variables $\Gamma_{t}$, the measure $\mathcal{Q}$ satisfies

$$
\mathcal{Q}\left[\Gamma_{t} \mathbb{1}_{g^{[a]} \leq t}\right]=\mathbb{P}\left[\Gamma_{t}\left(X_{t}-a\right)_{+}\right]
$$

Moreover, $\left(\left(X_{t}-a\right)_{+}\right)_{t \geq 0}$ is a submartingale of class $(\Sigma)$ and the $\sigma$-finite measure obtained by applying Theorem 1.1 to it is equal to $\mathcal{Q}$.

Proof. Let

$$
d_{t}^{[a]}:=\inf \left\{v>t: X_{v} \leq a\right\} .
$$

By the début theorem (for the natural conditions), $d_{t}^{[a]}$ is a stopping time. Now, for all $u>t$,

$$
\mathcal{Q}\left[\Gamma_{t} \mathbb{1}_{g \leq u, d_{t}^{[a]}>u}\right]=\mathbb{P}\left[\Gamma_{t} \mathbb{1}_{d_{t}^{[a]}>u} X_{u}\right] .
$$

One deduces, by using the decomposition of the submartingale $\left(X_{t}\right)_{t \geq 0}$, and by applying the martingale property to $\left(N_{t}\right)_{t \geq 0}$, that

$$
\begin{aligned}
\mathcal{Q}\left[\Gamma_{t} \mathbb{1}_{g \leq u, d_{t}^{[a]}>u}\right] & =\mathbb{P}\left[\Gamma_{t} \mathbb{1}_{d_{t}^{[a]}>u}\left(N_{u}+A_{u}\right)\right]=\mathbb{P}\left[\Gamma_{t} \mathbb{1}_{d_{t}^{[a]}>u}\left(N_{u}+A_{t}\right)\right] \\
& =\mathbb{P}\left[\Gamma_{t} \mathbb{1}_{d_{t}^{[a]}>u} A_{t}\right]+\mathbb{P}\left[\Gamma_{t} N_{u}\right]-\mathbb{P}\left[\Gamma_{t} \mathbb{1}_{d_{t}^{[a]} \leq u} N_{u}\right] \\
& =\mathbb{P}\left[\Gamma_{t} \mathbb{1}_{d_{t}^{[a]}>u} A_{t}\right]+\mathbb{P}\left[\Gamma_{t} N_{t}\right]-\mathbb{P}\left[\Gamma_{t} \mathbb{1}_{d_{t}^{[a]} \leq u} N_{d_{t}^{[a]}}\right] \\
& =\mathbb{P}\left[\Gamma_{t} \mathbb{1}_{d_{t}^{[a]}>u} A_{t}\right]+\mathbb{P}\left[\Gamma_{t} N_{t}\right]-\mathbb{P}\left[\Gamma_{t} \mathbb{1}_{d_{t}^{[a]} \leq u} X_{d_{t}^{[a]}}\right]+\mathbb{P}\left[\Gamma_{t} \mathbb{1}_{d_{t}^{[a]} \leq u} A_{t}\right] \\
& =\mathbb{P}\left[\Gamma_{t} X_{t}\right]-\mathbb{P}\left[\Gamma_{t} \mathbb{1}_{d_{t}^{[a]} \leq u} X_{\left.d_{t}^{[a]}\right] .}\right.
\end{aligned}
$$


In the above equalities we have used the fact that $A_{u}=A_{t}$ on the set $\left\{d_{t}^{[a]}>u\right\}$ and $A_{d_{t}^{[a]}}=A_{t}$ on $\left\{d_{t}^{[a]} \leq u\right\}$. Now, by right continuity, $d_{t}^{[a]}=t$ if $X_{t}<a$, and since $X$ has only positive jumps, for $X_{t} \geq a$ and $d_{t}^{[a]}<\infty, X_{d_{t}^{[a]}}=a$. One deduces that

$$
\mathcal{Q}\left[\Gamma_{t} \mathbb{1}_{g \leq u, d_{t}^{[a]}>u}\right]=\mathbb{P}\left[\Gamma_{t} X_{t}\right]-\mathbb{P}\left[\Gamma_{t} \mathbb{1}_{d_{t}^{[a]} \leq u}\left(X_{t} \wedge a\right)\right] .
$$

As $u \rightarrow \infty$, the event $\left\{g \leq u, d_{t}^{[a]}>u\right\}$ tends to $\left\{g^{[a]} \leq t\right\}$. Moreover, the event $\left\{d_{t}^{[a]} \leq u\right\}$ tends to $\left\{d_{t}^{[a]}<\infty\right\}$, which is almost sure under $\mathbb{P}$, since $A_{\infty}=\infty$. One deduces that

$$
\mathcal{Q}\left[\Gamma_{t} \mathbb{1}_{g^{[a]} \leq t}\right]=\mathbb{P}\left[\Gamma_{t} X_{t}\right]-\mathbb{P}\left[\Gamma_{t}\left(X_{t} \wedge a\right)\right]=\mathbb{P}\left[\Gamma_{t}\left(X_{t}-a\right)_{+}\right] .
$$

Now, from Lemma 2.1 in [6], $\left(\left(X_{t}-a\right)_{+}\right)_{t \geq 0}$ is also a nonnegative submartingale of class $(\Sigma)$. The supremum of its hitting times of zero is $g^{[a]}$. The formula (3.1) and the fact that $\left\{g^{[a]}<\infty\right\}$ holds $\mathcal{Q}$-almost everywhere (recall that $X_{t} \rightarrow \infty$ as $t \rightarrow \infty$, since $A_{\infty}=\infty \mathbb{P}$-almost surely) imply that $\mathcal{Q}$ is the $\sigma$-finite measure obtained from the submartingale $\left(\left(X_{t}-a\right)_{+}\right)_{t \geq 0}$.

In their study of Brownian penalizations, Najnudel, Roynette and Yor [11] introduce a particular class of nonnegative processes which converge $\mathcal{Q}$-almost everywhere to a $\mathcal{Q}$-integrable functional. Let us state a similar definition in our general framework:

Definition 3.1. Suppose that the assumptions of Theorem 1.1 are satisfied. We say that a process $\left(F_{t}\right)_{t \geq 0}$ belongs to the class $(\mathrm{C})$ if it is nonnegative, uniformly bounded, nonincreasing, càdlàg and adapted with respect to $\left(\mathcal{F}_{t}\right)_{t \geq 0}$, there exists $a>0$ such that for all $t \geq 0, F_{t}=F_{g^{[a]}}$ on the set $\left\{t \geq g^{[a]}\right\}$, and the decreasing limit of the process at infinity, denoted $F_{\infty}$, is $\mathcal{Q}$-integrable.

For example, the process $\left(F_{t}\right)_{t \geq 0}$ given by

$$
F_{t}=\varphi\left(A_{t}\right),
$$

where $\varphi: \mathbb{R}_{+} \rightarrow \mathbb{R}_{+}$is integrable and decreasing, is in the class (C), as also is

$$
F_{t}:=\exp \left(-\lambda A_{t}-\int_{0}^{t} q\left(X_{s}\right) d s\right),
$$

where $\lambda>0$ and $q$ is a measurable function from $\mathbb{R}_{+}$to $\mathbb{R}_{+}$, with compact support. When a process $\left(F_{t}\right)_{t \geq 0}$ is in the class $(\mathrm{C})$, the following proposition gives the behaviour of $\mathbb{P}\left[F_{t} X_{t}\right]$ as $t \rightarrow \infty$. 
Theorem 3.3. Suppose that the assumptions of Theorem 1.1 are satisfied, and that $A_{\infty}=\infty \mathbb{P}$-almost surely. Let $\left(F_{t}\right)_{t \geq 0}$ be a process in the class $(\mathrm{C})$. If $F_{g}$ is integrable with respect to $\mathcal{Q}$, then

$$
\mathbb{P}\left[F_{t} X_{t}\right] \underset{t \rightarrow \infty}{\longrightarrow} \mathcal{Q}\left[F_{\infty}\right]
$$

Proof. It is sufficient to prove

$$
\mathcal{Q}\left[F_{t} \mathbb{1}_{g \leq t}\right] \underset{t \rightarrow \infty}{\longrightarrow} \mathcal{Q}\left[F_{\infty}\right]
$$

Now, since the set $\left\{g^{[a]} \leq t\right\}$ is included in $\{g \leq t\}$, one can write

$$
\mathcal{Q}\left[F_{t} \mathbb{1}_{g \leq t}\right]=\mathcal{Q}\left[F_{t} \mathbb{1}_{g^{[a]} \leq t}\right]+\mathcal{Q}\left[F_{t} \mathbb{1}_{g \leq t<g^{[a]}}\right]
$$

Moreover

$$
\mathcal{Q}\left[F_{t} \mathbb{1}_{g^{[a]} \leq t}\right]=\mathcal{Q}\left[F_{\infty} \mathbb{1}_{g^{[a]} \leq t}\right] \underset{t \rightarrow \infty}{\longrightarrow} \mathcal{Q}\left[F_{\infty} \mathbb{1}_{g^{[a]}<\infty}\right]=\mathcal{Q}\left[F_{\infty}\right]
$$

The last equality is due to the fact that if $A_{\infty}=\infty \mathbb{P}$-almost surely, the process $\left(X_{t}\right)_{t \geq 0}$ tends $\mathcal{Q}$-almost everywhere to infinity with $t$. Hence, it is sufficient to prove that

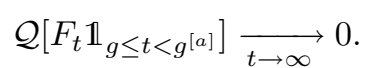

Now, $F_{t} \mathbb{1}_{g \leq t<g^{[a]}}$ is dominated by $F_{g}$, integrable with respect to $\mathcal{Q}$, and tends to zero $\mathcal{Q}$-almost surely as $t \rightarrow \infty$. By dominated convergence, we are done.

Remark. Let $\left(X_{t}\right)_{t \geq 0}$ be the absolute value of the Wiener process and let $F_{t}:=$ $\exp \left(-\lambda L_{t}\right)$, where $L_{t}$ is the local time of $\left(X_{t}\right)_{t \geq 0}$ at level 0 . The process $\left(F_{t}\right)_{t \geq 0}$ is in the class (C) and it is known (see [10]) that $L_{\infty}$ follows the Lebesgue measure on $\mathbb{R}_{+}$under $\mathcal{Q}$. Consequently,

$$
\mathbb{P}\left[\exp \left(-\lambda L_{t}\right) X_{t}\right] \underset{t \rightarrow \infty}{\longrightarrow} 1 / \lambda
$$

although

$$
\exp \left(-\lambda L_{t}\right) X_{t} \underset{t \rightarrow \infty}{\longrightarrow} 0
$$

$\mathbb{P}$-almost surely. Of course, due to the general feature of our results, the same result holds if one replaces $X_{t}$ by $\left|Y_{t}\right|^{\alpha-1}$, where $Y$ is a symmetric $\alpha$-stable Lévy process with index $\alpha \in(1,2)$, and $L_{t}$ would then stand for the local time of $Y$.

Here is another version of the same result (which does not involve the class (C)), which is in fact more powerful and useful:

Theorem 3.4. Suppose that the assumptions of Theorem 1.1 are satisfied, and that $A_{\infty}=\infty \mathbb{P}$-almost surely. Let $\left(F_{t}\right)_{t \geq 0}$ be a càdlàg, adapted, nonnegative 
process such that its limit $F_{\infty}$ exists $\mathcal{Q}$-almost everywhere. Suppose that there exists a $\mathcal{Q}$-integrable, nonnegative functional $H$ such that for all $t \geq 0$,

$$
F_{t} X_{t} \leq M_{t}(H)
$$

$\mathbb{P}$-almost surely. Then $F_{\infty}$ is $\mathcal{Q}$-integrable and

$$
\mathbb{P}\left[F_{t} X_{t}\right] \underset{t \rightarrow \infty}{\longrightarrow} \mathcal{Q}\left[F_{\infty}\right] .
$$

Proof. For all $t \geq 0$, one has $\mathcal{Q}$-almost everywhere

$$
F_{t} X_{t} \leq M_{t}(H) .
$$

Indeed, the event $\left\{F_{t} X_{t}>M_{t}(H)\right\}$ is $\mathcal{F}_{t}$-measurable and $\mathbb{P}$-negligible, and hence $\mathcal{Q}$-negligible. One deduces that $\mathbb{P}$-almost surely and $\mathcal{Q}$-almost everywhere, (3.2) is satisfied for all rationals $t \geq 0$, and so for all $t \geq 0$, since $\left(F_{t}\right)_{t \geq 0},\left(M_{t}(H)\right)_{t \geq 0}$ and $\left(X_{t}\right)_{t \geq 0}$ are càdlàg. By adding $e^{-A_{\infty}}$ to $H$, one can now suppose that $H>0$ and $M_{t}(H)>0$ for all $t$. Hence

$$
\mathbb{P}\left[F_{t} X_{t}\right]=\mathcal{M}^{H}\left[\frac{F_{t} X_{t}}{M_{t}(H)}\right] .
$$

Now, uniformly in $t$,

$$
\frac{F_{t} X_{t}}{M_{t}(H)} \leq 1+\infty \cdot \mathbb{1}_{\exists t \geq 0, F_{t} X_{t}>M_{t}(H)},
$$

which is $\mathcal{M}^{H}$-integrable, since $\mathcal{M}^{H}$ is a finite measure and the event $\{\exists t \geq 0$, $\left.F_{t} X_{t}>M_{t}(H)\right\}$ is $\mathcal{Q}$-negligible, and so $\mathcal{M}^{H}$-negligible, In particular:

$$
\mathbb{P}\left[F_{t} X_{t}\right] \leq \mathcal{M}^{H}\left[1+\infty \cdot \mathbb{1}_{\exists t \geq 0, F_{t} X_{t}>M_{t}(H)}\right]=\mathcal{Q}[H]<\infty .
$$

Moreover, $\mathcal{Q}$-almost everywhere,

$$
\frac{F_{t} X_{t}}{M_{t}(H)} \underset{t \rightarrow \infty}{\longrightarrow} \frac{F_{\infty}}{H} .
$$

By dominated convergence,

$$
\mathbb{P}\left[F_{t} X_{t}\right] \underset{t \rightarrow \infty}{\longrightarrow} \mathcal{M}^{H}\left[\frac{F_{\infty}}{H}\right]=\mathcal{Q}\left[F_{\infty}\right] .
$$

Since $\mathbb{P}\left[F_{t} X_{t}\right] \leq \mathcal{Q}[H]$ for all $t \geq 0$, one deduces that $\mathcal{Q}\left[F_{\infty}\right] \leq \mathcal{Q}[H]<\infty$.

We now illustrate how the above result can be used. Let $f: \mathbb{R}_{+} \rightarrow \mathbb{R}_{+}$be an integrable function. From the identity (1.4) defining the martingale $\left(M_{t}^{f}\right)_{t \geq 0}$, and using the fact that $A_{\infty}=\infty \mathbb{P}$-almost surely, we obtain

$$
f\left(A_{t}\right) X_{t} \leq M_{t}^{f} .
$$


Consequently, the above proposition applies to the case $F_{t}=f\left(A_{t}\right)$, where $f$ : $\mathbb{R}_{+} \rightarrow \mathbb{R}_{+}$is an integrable function. It also obviously applies to any function $F_{t}$ which satisfies $F_{t} \leq f\left(A_{t}\right)$ for some integrable $f: \mathbb{R}_{+} \rightarrow \mathbb{R}_{+}$, for instance, $F_{t}=$ $G_{t} f\left(A_{t}\right)$ where $G_{t}$ is a bounded càdlàg $\mathcal{F}_{t}$-measurable process and $f: \mathbb{R}_{+} \rightarrow \mathbb{R}_{+}$ is integrable; in particular it applies to $F_{t}=f\left(A_{t}\right) \exp \left(-\int_{0}^{t} q\left(X_{s}\right) d s\right)$, where $q$ is a measurable function from $\mathbb{R}_{+}$to $\mathbb{R}_{+}$.

We are now able to state two universal penalization results:

Theorem 3.5. Suppose that the assumptions of Theorem 1.1 are satisfied, and that $A_{\infty}=\infty \mathbb{P}$-almost surely. Let $\left(F_{t}\right)_{t \geq 0}$ be a process in the class $(\mathrm{C})$ such that $F_{g}$ is integrable with respect to $\mathcal{Q}$ and $F_{\infty}$ is not $\mathcal{Q}$-almost everywhere equal to zero. Then, for $t$ sufficiently large, $0<\mathbb{P}\left[F_{t} X_{t}\right]<\infty$, and one can define a measure $\mathbb{Q}_{t}$ by

$$
\mathbb{Q}_{t}=\frac{F_{t} X_{t}}{\mathbb{P}\left[F_{t} X_{t}\right]} \mathbb{P} .
$$

Moreover, there exists a probability measure $\mathbb{Q}_{\infty}$ which can be considered as the weak limit of $\mathbb{Q}_{t}$ as $t \rightarrow \infty$, in the following sense: for all $s \geq 0$ and all events $\Lambda_{s} \in \mathcal{F}_{s}$,

$$
\mathbb{Q}_{t}\left[\Lambda_{s}\right] \underset{t \rightarrow \infty}{\longrightarrow} \mathbb{Q}_{\infty}\left[\Lambda_{s}\right] .
$$

The measure $\mathbb{Q}_{\infty}$ is absolutely continuous with respect to $\mathcal{Q}$ :

$$
\mathbb{Q}_{\infty}=\frac{F_{\infty}}{\mathcal{Q}\left[F_{\infty}\right]} \mathcal{Q}, \quad \text { where } 0<\mathcal{Q}\left[F_{\infty}\right]<\infty .
$$

Proof. The integrability of $F_{\infty}$ under $\mathcal{Q}$ is an immediate consequence of the integrability of $F_{g}$, and $\mathcal{Q}\left[F_{\infty}\right]>0$ because $F_{\infty}$ is not $\mathcal{Q}$-almost everywhere equal to zero. Moreover, for all $t \geq 0, F_{t}$ is uniformly bounded and $X_{t}$ is $\mathbb{P}$-integrable, which implies that $\mathbb{P}\left[F_{t} X_{t}\right]$ is finite. On the other hand, by Theorem 3.3,

$$
\mathbb{P}\left[F_{t} X_{t}\right] \underset{t \rightarrow \infty}{\longrightarrow} \mathcal{Q}\left[F_{\infty}\right]>0,
$$

and so $\mathbb{P}\left[F_{t} X_{t}\right]>0$ for $t$ large enough. Now, for $t>s$,

$$
\mathbb{P}\left[F_{t} \mathbb{1}_{\Lambda_{s}} X_{t}\right]=\mathcal{Q}\left[F_{t} \mathbb{1}_{\Lambda_{s}} \mathbb{1}_{g \leq t}\right],
$$

where, by the arguments in the proof of Theorem 3.3,

$$
\mathcal{Q}\left[F_{t} \mathbb{1}_{\Lambda_{s}} \mathbb{1}_{g \leq t}\right] \underset{t \rightarrow \infty}{\longrightarrow} \mathcal{Q}\left[F_{\infty} \mathbb{1}_{\Lambda_{s}}\right] .
$$

Combining this with (3.3) completes the proof of the theorem.

Theorem 3.6. Suppose that the assumptions of Theorem 1.1 are satisfied, and that $A_{\infty}=\infty \mathbb{P}$-almost surely. Let $\left(F_{t}\right)_{t \geq 0}$ be a càdlàg, adapted, nonnegative 
process such that its limit $F_{\infty}$ exists $\mathcal{Q}$-almost everywhere and is not $\mathcal{Q}$-almost everywhere equal to zero. Suppose that there exists a $\mathcal{Q}$-integrable, nonnegative functional $H$ such that for all $t \geq 0$,

$$
F_{t} X_{t} \leq M_{t}(H)
$$

$\mathbb{P}$-almost surely. Then, for $t$ sufficiently large, $0<\mathbb{P}\left[F_{t} X_{t}\right]<\infty$ and one can define a measure $\mathbb{Q}_{t}$ by

$$
\mathbb{Q}_{t}=\frac{F_{t} X_{t}}{\mathbb{P}\left[F_{t} X_{t}\right]} \mathbb{P} .
$$

Moreover, there exists a probability measure $\mathbb{Q}_{\infty}$ which can be considered as the weak limit of $\mathbb{Q}_{t}$ as $t \rightarrow \infty$, in the following sense: for all $s \geq 0$ and all $\Lambda_{s} \in \mathcal{F}_{s}$,

$$
\mathbb{Q}_{t}\left[\Lambda_{s}\right] \underset{t \rightarrow \infty}{\longrightarrow} \mathbb{Q}_{\infty}\left[\Lambda_{s}\right] .
$$

The measure $\mathbb{Q}_{\infty}$ is absolutely continuous with respect to $\mathcal{Q}$ :

$$
\mathbb{Q}_{\infty}=\frac{F_{\infty}}{\mathcal{Q}\left[F_{\infty}\right]} \mathcal{Q}, \quad \text { where } 0<\mathcal{Q}\left[F_{\infty}\right]<\infty .
$$

Proof. Since for all $t \geq 0, F_{t} X_{t} \leq M_{t}(H) \mathbb{P}$-almost surely, one has

$$
\mathbb{P}\left[F_{t} X_{t}\right] \leq \mathbb{P}\left[M_{t}(H)\right]=\mathcal{Q}[H]<\infty .
$$

On the other hand, by Theorem 3.4,

$$
\mathbb{P}\left[F_{t} X_{t}\right] \underset{t \rightarrow \infty}{\longrightarrow} \mathcal{Q}\left[F_{\infty}\right] \in(0, \infty),
$$

which implies that $\mathbb{P}\left[F_{t} X_{t}\right]>0$ for $t$ large enough. Moreover, by applying Theorem 3.4 to the family of functionals $\left(F_{t} \mathbb{1}_{\Lambda_{s}} \mathbb{1}_{t \geq s}\right)_{t \geq 0}$, one deduces that

$$
\mathbb{P}\left[F_{t} \mathbb{1}_{\Lambda_{s}} X_{t}\right] \underset{t \rightarrow \infty}{\longrightarrow} \mathcal{Q}\left[\mathbb{1}_{\Lambda_{s}} F_{\infty}\right]
$$

Combining this with (3.4) completes the proof of the theorem.

Remark. To obtain penalization results which do not necessarily involve $X_{t}$, e.g. of the form $F_{t}=f\left(A_{t}\right)$, we need to find an equivalent for $\mathbb{P}\left[F_{t}\right]$. Unfortunately, we are not able to give such an estimate in the general case; however, if $\left(X_{t}\right)_{t \geq 0}$ is a diffusion satisfying some technical conditions, this problem is solved in the companion paper [9], and we deduce a penalization theorem, generalizing results given in [11]. 


\section{§4. A new decomposition of nonnegative supermartingales}

The following proposition gives a general decomposition of any nonnegative, càdlàg supermartingale, involving a uniform martingale and a martingale of the form $\left(M_{t}(F)\right)_{t \geq 0}$. This decomposition generalizes a result obtained in [11] (Theorem $1.2 .5)$.

Theorem 4.1. Suppose that the assumptions of Theorem 1.1 are satisfied, and that $A_{\infty}=\infty \mathbb{P}$-almost surely. Let $\left(Z_{t}\right)_{t \geq 0}$ be a nonnegative, càdlàg $\mathbb{P}$-supermartingale. Denote by $Z_{\infty}$ the $\mathbb{P}$-almost sure limit of $Z_{t}$ as $t \rightarrow \infty$. Then, $\mathcal{Q}$-almost everywhere, the quotient $Z_{t} / X_{t}$ is well-defined for $t$ large enough and converges, as $t \rightarrow \infty$, to a limit $z_{\infty}$, integrable with respect to $\mathcal{Q}$, and $\left(Z_{t}\right)_{t \geq 0}$ decomposes as

$$
\left(Z_{t}=M_{t}\left(z_{\infty}\right)+\mathbb{P}\left[Z_{\infty} \mid \mathcal{F}_{t}\right]+\xi_{t}\right)_{t \geq 0}
$$

where $\left(\mathbb{P}\left[Z_{\infty} \mid \mathcal{F}_{t}\right]\right)_{t \geq 0}$ denotes a càdlàg version of the conditional expectation of $Z_{\infty}$ with respect to $\mathcal{F}_{t}$, and $\left(\xi_{t}\right)_{t \geq 0}$ is a nonnegative, càdlàg $\mathbb{P}$-supermartingale, such that:

- $Z_{\infty} \in L_{+}^{1}(\mathcal{F}, \mathbb{P})$, hence $\mathbb{P}\left[Z_{\infty} \mid \mathcal{F}_{t}\right]$ converges $\mathbb{P}$-almost surely and in $L^{1}(\mathcal{F}, \mathbb{P})$ towards $Z_{\infty}$.

- $\frac{\mathbb{P}\left[Z_{\infty} \mid \mathcal{F}_{t}\right]+\xi_{t}}{X_{t}} \underset{t \rightarrow \infty}{\longrightarrow} 0 \mathcal{Q}$-almost everywhere.

- $M_{t}\left(z_{\infty}\right)+\xi_{t} \underset{t \rightarrow \infty}{\longrightarrow} 0 \mathbb{P}$-almost surely.

Moreover, the decomposition is unique in the following sense: Let $z_{\infty}^{\prime}$ be a $\mathcal{Q}$ integrable, nonnegative functional, $Z_{\infty}^{\prime}$ a $\mathbb{P}$-integrable, nonnegative random variable, $\left(\xi_{t}^{\prime}\right)_{t \geq 0}$ a càdlàg, nonnegative $\mathbb{P}$-supermartingale, and suppose that for all $t \geq 0$

$$
Z_{t}=M_{t}\left(z_{\infty}^{\prime}\right)+\mathbb{P}\left[Z_{\infty}^{\prime} \mid \mathcal{F}_{t}\right]+\xi_{t}^{\prime}
$$

Under these assumptions, if as $t \rightarrow \infty, \xi_{t}^{\prime}$ tends $\mathbb{P}$-almost surely to zero and $\xi_{t}^{\prime} / X_{t}$ tends $\mathcal{Q}$-almost everywhere to zero, then $z_{\infty}^{\prime}=z_{\infty} \mathcal{Q}$-almost everywhere, $Z_{\infty}^{\prime}=Z_{\infty}$ $\mathbb{P}$-almost surely, and $\xi^{\prime}$ is $\mathbb{P}$-indistinguishable from $\xi$.

Proof. Let $H:=e^{-A_{\infty}}$. Since $Z$ is a càdlàg $\mathbb{P}$-supermartingale, it is easy to deduce that $\left(Z_{t} / M_{t}(H)\right)_{t \geq 0}$ is a càdlàg supermartingale with respect to $\widetilde{\mathcal{M}}^{H}:=$ $\mathcal{M}^{H} / \mathcal{M}^{H}(1)$. Hence, it converges $\widetilde{\mathcal{M}}^{H}$-almost surely to a limit $\zeta$. Since $M_{t}(H) / X_{t}$ converges $\widetilde{\mathcal{M}}^{H}$-a.s. to $H, Z_{t} / X_{t}$ converges $\widetilde{\mathcal{M}}^{H}$-a.s., and hence $\mathcal{Q}$-almost everywhere, to $z_{\infty}=\zeta H$. Moreover, since $\zeta$ is the $\widetilde{\mathcal{M}}^{H}$-a.s. limit of the $\widetilde{\mathcal{M}}^{H}$ supermartingale $\left(Z_{t} / M_{t}(H)\right)_{t \geq 0}$, one has

$$
\mathcal{Q}\left[z_{\infty}\right]=\mathcal{M}^{H}[\zeta] \leq \mathcal{M}^{H}\left[Z_{0} / M_{0}(H)\right]<\infty
$$


Since $z_{\infty}$ is $\mathcal{Q}$-integrable, $\left(M_{t}\left(z_{\infty}\right)\right)_{t \geq 0}$ is well-defined. Now, for all nonnegative, $\mathcal{F}_{t}$-measurable functionals $\Gamma_{t}$,

$$
\begin{aligned}
\mathcal{Q}\left[\Gamma_{t} z_{\infty}\right] & =\mathcal{Q}\left[\Gamma_{t} \lim _{u \rightarrow \infty} \frac{Z_{u}}{X_{u}}\right]=\mathcal{Q}\left[\Gamma_{t} \lim _{u \rightarrow \infty} \frac{Z_{u}}{X_{u}} \mathbb{1}_{g \leq u}\right] \\
& \leq \liminf _{u \rightarrow \infty} \mathcal{Q}\left[\Gamma_{t} \frac{Z_{u}}{X_{u}} \mathbb{1}_{g \leq u}\right]=\liminf _{u \rightarrow \infty} \mathbb{P}\left[\Gamma_{t} \frac{Z_{u}}{X_{u}} X_{u}\right] \\
& \leq \liminf _{u \rightarrow \infty} \mathbb{P}\left[\Gamma_{t} Z_{u}\right] \leq \mathbb{P}\left[\Gamma_{t} Z_{t}\right] .
\end{aligned}
$$

Thus for all $t \geq 0, M_{t}\left(z_{\infty}\right) \leq Z_{t} \mathbb{P}$-a.s., which implies that the process $\left(M_{t}\left(z_{\infty}\right) \wedge Z_{t}\right)_{t \geq 0}$ is a càdlàg and adapted modification of $\left(M_{t}\left(z_{\infty}\right)\right)_{t \geq 0}$. Since $\left(M_{t}\left(z_{\infty}\right)\right)_{t \geq 0}$ is only defined up to càdlàg modifications (which are indistinguishable from each other), one can replace $\left(M_{t}\left(z_{\infty}\right)\right)_{t \geq 0}$ by $\left(M_{t}\left(z_{\infty}\right) \wedge Z_{t}\right)_{t \geq 0}$, and therefore suppose that for all $t \geq 0, M_{t}\left(z_{\infty}\right) \leq Z_{t}$ everywhere. Note that if $\left(Z_{t}\right)_{t \geq 0}$ is supposed to be uniformly integrable, then so is $\left(M_{t}\left(z_{\infty}\right)\right)_{t \geq 0}$, and since it tends $\mathbb{P}$-almost surely to zero, it is $\mathbb{P}$-almost surely identically zero. This implies that $z_{\infty}=0 \mathcal{Q}$-almost everywhere. Now, going back to the general case, let us define, for all $t \geq 0$,

$$
\widetilde{Z}_{t}:=Z_{t}-M_{t}\left(z_{\infty}\right)
$$

Since $\left(M_{t}\left(z_{\infty}\right)\right)_{t \geq 0}$ is a càdlàg $\mathbb{P}$-martingale, the process $\left(\widetilde{Z}_{t}\right)_{t \geq 0}$ is a càdlàg, nonnegative $\mathbb{P}$-supermartingale. Moreover, $M_{t}\left(z_{\infty}\right)$ tends $\mathbb{P}$-almost surely to zero as $t \rightarrow \infty$, hence

$$
\widetilde{Z}_{t} \underset{t \rightarrow \infty}{\longrightarrow} Z_{\infty}
$$

$\mathbb{P}$-almost surely. Now, since $\left(\widetilde{Z}_{t}\right)_{t \geq 0}$ is a nonnegative supermartingale and $Z_{\infty} \geq 0$ $\mathbb{P}$-almost surely, we obtain, for all $t \geq 0$,

$$
0 \leq \mathbb{P}\left[Z_{\infty} \mid \mathcal{F}_{t}\right] \leq \widetilde{Z}_{t}
$$

$\mathbb{P}$-almost surely. Hence, $\left(\left(\mathbb{P}\left[Z_{\infty} \mid \mathcal{F}_{t}\right]\right)_{+} \wedge \widetilde{Z}_{t}\right)_{t \geq 0}$ is a càdlàg version of $\left(\mathbb{P}\left[Z_{\infty} \mid \mathcal{F}_{t}\right]\right)_{t \geq 0}$ and one can suppose that (4.1) holds everywhere. Now, let us write, for all $t \geq 0$,

$$
\xi_{t}:=\widetilde{Z}_{t}-\mathbb{P}\left[Z_{\infty} \mid \mathcal{F}_{t}\right] .
$$

This is a nonnegative, càdlàg supermartingale tending $\mathbb{P}$-a.s. to zero as $t \rightarrow \infty$. On the other hand, $\mathcal{Q}$-almost everywhere,

$$
\lim _{t \rightarrow \infty} \frac{\xi_{t}}{X_{t}}=\lim _{t \rightarrow \infty} \frac{\widetilde{Z}_{t}}{X_{t}}=z_{\infty}-z_{\infty}=0 .
$$

Here, the first equality is due to the fact that $\left(\mathbb{P}\left[Z_{\infty} \mid \mathcal{F}_{t}\right] / X_{t}\right)_{t \geq 0}$ tends to zero $\mathcal{Q}$-almost everywhere, by the remark made above on the case where $\left(Z_{t}\right)_{t \geq 0}$ is 
uniformly integrable. The uniqueness of the decomposition is very easy to check: since $M_{t}\left(z_{\infty}^{\prime}\right)$ and $\xi_{t}^{\prime}$ tend $\mathbb{P}$-almost surely to zero as $t \rightarrow \infty, Z_{t}$ tends $\mathbb{P}$-almost surely to $Z_{\infty}^{\prime}$ and so $Z_{\infty}^{\prime}=Z_{\infty}$. Similarly, since $\xi_{t}^{\prime} / X_{t}$ and any càdlàg version of $\mathbb{P}\left[Z_{\infty}^{\prime} \mid \mathcal{F}_{t}\right] / X_{t}$ tend to zero $\mathcal{Q}$-almost everywhere, $Z_{t} / X_{t}$ tends to $z_{\infty}^{\prime}$, which is $\mathcal{Q}$-almost everywhere equal to $z_{\infty}$. One now deduces that for all $t \geq 0, \xi_{t}^{\prime}=\xi_{t}$ $\mathbb{P}$-almost surely, and since $\xi$ and $\xi^{\prime}$ are càdlàg, they are indistinguishable, which proves the uniqueness of the decomposition.

As in [11], we can deduce, from Theorem 4.1, the following characterization of the martingales of the form $\left(M_{t}(F)\right)_{t \geq 0}$ :

Corollary 4.1. Suppose that the assumptions of Theorem 1.1 are satisfied, and that $A_{\infty}=\infty \mathbb{P}$-almost surely. Then a càdlàg, nonnegative $\mathbb{P}$-martingale $\left(Z_{t}\right)_{t \geq 0}$ has the form $\left(M_{t}(F)\right)_{t \geq 0}$ for a nonnegative, $\mathcal{Q}$-integrable functional $F$ if and only if

$$
\mathbb{P}\left[Z_{0}\right]=\mathcal{Q}\left(\lim _{t \rightarrow \infty} \frac{Z_{t}}{X_{t}}\right) .
$$

Note that, by Theorem 4.1, the limit above necessarily exists $\mathcal{Q}$-almost everywhere.

Proof. By Theorem 4.1, one can write the decomposition

$$
Z_{t}=M_{t}\left(z_{\infty}\right)+\mathbb{P}\left[Z_{\infty} \mid \mathcal{F}_{t}\right]+\xi_{t} .
$$

Note that in this situation, $\left(\xi_{t}\right)_{t \geq 0}$ is a nonnegative martingale. One has

$$
\mathbb{P}\left[Z_{0}\right]=\mathbb{P}\left[M_{0}\left(z_{\infty}\right)\right]+\mathbb{P}\left[\mathbb{P}\left[Z_{\infty} \mid \mathcal{F}_{0}\right]\right]+\mathbb{P}\left[\xi_{0}\right]=\mathcal{Q}\left[z_{\infty}\right]+\mathbb{P}\left[Z_{\infty}\right]+\mathbb{P}\left[\xi_{0}\right] .
$$

Now, the equation (4.2) is satisfied if and only if

$$
\mathbb{P}\left[Z_{0}\right]=\mathcal{Q}\left[z_{\infty}\right] .
$$

If this condition holds, one has

$$
\mathbb{P}\left[Z_{\infty}\right]=\mathbb{P}\left[\xi_{0}\right]=0,
$$

and then, for all $t \geq 0$,

$$
\mathbb{P}\left[Z_{\infty} \mid \mathcal{F}_{t}\right]+\xi_{t}=0
$$

almost surely. Hence, the martingale $\left(Z_{t}\right)_{t \geq 0}$ is a càdlàg modification of $\left(M_{t}\left(z_{\infty}\right)\right)_{t \geq 0}$. Since $\left(M_{t}\left(z_{\infty}\right)\right)_{t \geq 0}$ is only defined up to càdlàg modification, one can suppose that $\left(Z_{t}\right)_{t \geq 0}$ coincides with $\left(M_{t}\left(z_{\infty}\right)\right)_{t \geq 0}$. On the other hand, if $\left(Z_{t}\right)_{t \geq 0}$ has the form $\left(M_{t}(F)\right)_{t \geq 0}$, by uniqueness of the decomposition given in Proposition $4.1, F=z_{\infty} \mathcal{Q}$-almost everywhere, which implies that $\mathbb{P}\left[Z_{0}\right]=\mathcal{Q}\left[z_{\infty}\right]$, and so (4.2) is satisfied. 
Remark. Suppose that, in Theorem 4.1, $\left(Z_{t}\right)_{t \geq 0}$ is a nonnegative martingale. Since the space has property $(\mathrm{NP})$, there exists a unique finite measure $\mathbb{Q}_{Z}$ on $(\Omega, \mathcal{F})$ such that for all $t \geq 0$, its restriction to $\mathcal{F}_{t}$ has density $Z_{t}$ with respect to $\mathbb{P}$. If one writes the decomposition

$$
Z_{t}=M_{t}\left(z_{\infty}\right)+\mathbb{P}\left[Z_{\infty} \mid \mathcal{F}_{t}\right]+\xi_{t}
$$

one deduces

$$
\mathbb{Q}_{Z}=z_{\infty} \mathcal{Q}+Z_{\infty} \mathbb{P}+\mathbb{Q}_{\xi},
$$

where the restriction of $\mathbb{Q}_{\xi}$ to $\mathcal{F}_{t}$ has density $\xi_{t}$ with respect to $\mathbb{P}$. By the RadonNikodym theorem, one has a decomposition

$$
\mathbb{Q}_{\xi}=\xi^{\prime} \mathbb{P}+\mathbb{Q}_{\xi}^{\prime},
$$

where $\mathbb{Q}_{\xi}^{\prime}$ is singular with respect to $\mathbb{P}$. Now, if for $t \geq 0, \xi_{t}^{\prime}$ is the density, with respect to $\mathbb{P}$, of the restriction of $\xi^{\prime} \mathbb{P}$ to $\mathcal{F}_{t}$, then for all $t \geq 0, \xi_{t}^{\prime} \leq \xi_{t} \mathbb{P}$-almost surely. Moreover, if $\left(\xi_{t}^{\prime}\right)_{t \geq 0}$ is supposed to be càdlàg, then almost surely, $\xi_{t}^{\prime} \leq \xi_{t}$ for all $t \geq 0$. By taking the $\mathbb{P}$-almost sure limit for $t$ going to infinity, one deduces that $\xi^{\prime}=0 \mathbb{P}$-almost surely, and therefore $\mathbb{Q}_{\xi}=\mathbb{Q}_{\xi}^{\prime}$ is singular with respect to $\mathbb{P}$. One can also decompose $\mathbb{Q}_{\xi}$ as

$$
\mathbb{Q}_{\xi}=\xi^{\prime \prime} \mathcal{Q}+\mathbb{Q}_{\xi}^{\prime \prime}
$$

where $\mathbb{Q}_{\xi}^{\prime \prime}$ is singular with respect to $\mathcal{Q}$. Now, for all $t \geq 0$, one has, $\mathbb{P}$-almost surely, and hence $\mathcal{Q}$-almost everywhere, $M_{t}\left(\xi^{\prime \prime}\right) \leq \xi_{t}$. Since $\left(M_{t}\left(\xi^{\prime \prime}\right)\right)_{t \geq 0}$ and $\left(\xi_{t}\right)_{t \geq 0}$ are right-continuous, one deduces that $\mathcal{Q}$-almost everywhere, $M_{t}\left(\xi^{\prime \prime}\right) \leq \xi_{t}$ for all $t \geq 0$. Since $\mathcal{Q}$-almost everywhere, $M_{t}\left(\xi^{\prime \prime}\right) / X_{t}$ tends to $\xi^{\prime \prime}$ as $t \rightarrow \infty$, and $\xi_{t} / X_{t}$ tends to zero, one has $\xi^{\prime \prime}=0 \mathcal{Q}$-almost everywhere and $\mathbb{Q}_{\xi}=\mathbb{Q}_{\xi}^{\prime \prime}$ is singular with respect to $\mathcal{Q}$. Hence, we have obtained a decomposition of $\mathbb{Q}_{Z}$ into three parts:

- A part which is absolutely continuous with respect to $\mathbb{P}$.

- A part which is absolutely continuous with respect to $\mathcal{Q}$.

- A part which is singular with respect to $\mathbb{P}$ and $\mathcal{Q}$.

This decomposition is unique, as a consequence of uniqueness of the RadonNikodym decomposition (recall that $\mathbb{P}$ and $\mathcal{Q}$ are mutually singular, since $A_{\infty}=\infty$ $\mathbb{P}$-almost surely, and $A_{\infty}<\infty \mathcal{Q}$-almost everywhere). This uniqueness can be compared with the uniqueness of the decomposition of the martingale $\left(Z_{t}\right)_{t \geq 0}$ given in Proposition 4.1. 


\section{References}

[1] J. Azéma, P.-A. Meyer and M. Yor, Martingales relatives, in Séminaire de probabilités, XXVI, Lecture Notes in Math. 1526, Springer, 1992, 307-321. Zbl 0765.60037 MR 1232000

[2] J. Azéma and M. Yor, Sur les zéros des martingales continues, in Séminaire de probabilités, XXVI, Lecture Notes in Math. 1526, Springer, 1992, 248-306. Zbl 0765.60038 MR 1231999

[3] A. Bentata and M. Yor, From Black-Scholes and Dupire formulae to last passage times of local martingales. Part A: The infinite time horizon (2008).

[4] J. Bertoin, Lévy processes, Cambridge Univ. Press, 1996. Zbl 0861.60003 MR 1406564

[5] K. Bichteler, Stochastic integration with jumps, Cambridge Univ. Press, 2002. Zbl 1189.60004 MR 1906715

[6] P. Cheridito, A. Nikeghbali and E. Platen, Processes of the class sigma, last zero and drawdown processes (2009).

[7] D. Madan, B. Roynette and M. Yor, From Black-Scholes formula, to local times and last passage times for certain submartingales, Prépublication IECN 2008/14 (2008).

[8] J. Najnudel and A. Nikeghbali, A new kind of augmentation of filtrations, ESAIM Probab. Statist. 15 (2011), S39-S57.

[9] _ On penalisation results related with a remarkable class of submartingales, arXiv:0911.4365 (2009).

[10] ping theorem, arXiv:0906.1782 (2009).

[11] J. Najnudel, B. Roynette and M. Yor, A global view of Brownian penalisations, MSJ Memoirs 19 (2009). Zbl 1180.60004 MR 2528440

[12] A. Nikeghbali, A class of remarkable submartingales, Stochastic Process. Appl. 116 (2006), 917-938. Zbl 1104.60018 MR 2254665

[13] K.-R. Parthasarathy, Probability measures on metric spaces, Academic Press, New York, 1967. Zbl 0153.19101 MR 2169627

[14] B. Roynette, P. Vallois and M. Yor, Some penalisations of the Wiener measure, Japan J. Math. 1 (2006), 263-290. Zbl 1160.60315 MR 2261065

[15] P. Salminen and M. Yor, Tanaka formula for symmetric Lévy processes, in Séminaire de Probabilités, XL, Lecture Notes in Math. 1899, Springer, 2007, 265-285. Zbl 1129.60043 MR 2409011

[16] K. Yano, Y. Yano and M. Yor, Penalising symmetric stable Lévy paths, J. Math. Soc. Japan 61 (2009), 757-798. Zbl 1180.60008 MR 2552915

[17] M. Yor, Les inégalités de sous-martingales, comme conséquences de la relation de domination, Stochastics 3 (1979), 1-15. Zbl 0437.60038 MR 0546696 